Int Arch Allergy Immunol 2021;182:94-105

DOI: $10.1159 / 000510536$
Received: April 28, 2020

Accepted: July 29, 2020

Published online: September 24, 2020

\title{
The Gut Microbiome of Adults with Allergic Rhinitis Is Characterised by Reduced Diversity and an Altered Abundance of Key Microbial Taxa Compared to Controls
}

\author{
Annabelle M. Watts ${ }^{a}$ Nicholas P. West ${ }^{a, b}$ Ping Zhang ${ }^{b}$ Peter K. Smith ${ }^{c, d}$ \\ Allan W. Cripps ${ }^{b, c}$ Amanda J. Cox ${ }^{a, b}$

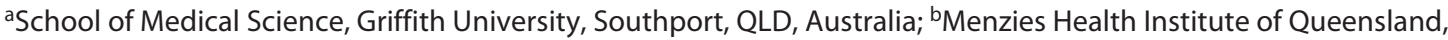 \\ Griffith University, Southport, QLD, Australia; 'School of Medicine, Griffith University, Southport, QLD, Australia; \\ ${ }^{\mathrm{d}}$ Queensland Allergy Services, Southport, QLD, Australia
}

\section{Keywords}

Microbiome $\cdot$ Allergic rhinitis $\cdot 16 \mathrm{~s}$ rRNA gene $\cdot$ Species richness · Gastrointestinal

\begin{abstract}
Introduction: Unique gut microbial colonisation patterns are associated with the onset of allergic disease in infants; however, there is insufficient evidence to determine if aberrant microbial composition patterns persist in adult allergic rhinitis (AR) sufferers. Objective: To compare the gut microbiome composition between adult AR sufferers and controls. Methods: Gut microbial composition in stool samples was compared between 57 adult AR sufferers (39.06 \pm 13.29 years) and 23 controls (CG; $36.55 \pm 10.51$ years) via next-generation sequencing of the V3-V4 hypervariable regions of the 16S rRNA gene. Taxonomic classification and identity assignment was performed using a reference-based approach with the NCBI database of $16 \mathrm{~S}$ rRNA gene sequences. $\boldsymbol{R e}$ sults: Species richness determined via the Shannon index was significantly reduced in the AR cohort compared to the CG (4.35 \pm 0.59 in AR vs. $4.65 \pm 0.55$ in CG, $p=0.037)$; trends for reductions in operational taxonomic unit (OTU) counts, inverse Simpson, and $\mathrm{CHAO} 1$ diversity indices were also not-
\end{abstract}

ed. Bacteroidetes $(p=0.014)$ was significantly more abundant in the AR group than in the CG. In contrast, the Firmicutes phylum was significantly less abundant in the $A R$ group than in the CG $(p=0.006)$. An increased abundance of Parabacteroides $(p=0.008)$ and a reduced abundance of $O x$ alobacter $(p=0.001)$ and Clostridiales $(p=0.005)$ were also observed in the AR cohort compared to the CG. Conclusion: Adult AR sufferers have a distinct gut microbiome profile, marked by a reduced microbial diversity and altered abundance of certain microbes compared to controls. The results of this study provide evidence that unique gut microbial patterns occur in AR sufferers in adulthood and warrant further examination in the form of mechanistic studies.

(c) 2020 S. Karger AG, Basel

\section{Introduction}

The gastrointestinal microbiota plays an important role in the development and regulation of local and systemic immunity. Indeed, several immune-mediated conditions [1-7], including allergic disease [8-11], have been

Edited by: A. Haczku, Sacramento, CA. karger@karger.com

www.karger.com/iaa

Karger
(C) 2020 S. Karger AG, Basel

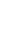

\footnotetext{
Allan W. Cripps

School of Medicine, Menzies Health Institute Queensland, Griffith University Parklands Drive

Southport, QLD 4215 (Australia)

allan.cripps@griffith.edu.au
} 
associated with abnormal gut microbiome composition. Allergic rhinitis (AR) is the most prevalent allergic disease and is characterised by a T helper (Th) 2 polarised response, which promotes IgE-mediated inflammation in the nasal mucosa following allergen exposure. Polarisation of the Th2 phenotype develops in utero and persists in neonates at birth $[12,13]$. Immune homeostasis and maturation towards a Th1 phenotype is dependent on colonisation of the gut with commensal microbes [14]. Data generated from germ-free mouse models have shown that commensal gut microbes regulate Th2 responses [15], with recent evidence suggesting this occurs via induction of enteric Th17 and T regulatory cells [16].

The relationship between the gut microbial composition and the onset of allergic disease has been extensively studied in paediatric populations and has been reviewed elsewhere [11]. A consistent finding in these paediatric studies is that lower microbial diversity occurs in the intestine of allergic infants than in non-allergic infants. While differences in microbial composition between allergic and non-allergic infants have been reported, a consensus of an "allergic microbiome profile" cannot be reached. Heterogeneity in study design features, including allergic disease classification, age of subjects, and microbiome identification tools used, complicate our understanding of the microbiome profile of allergic infants. Furthermore, the microbiome profile of allergic infants has been shown to differ by time of sample collection, where samples collected before the onset of allergic disease differ from samples collected during active disease. These data suggest that there is a time- or age-related effect on the development of gut microbiome, relative to atopy.

The gut microbiome is known to undergo rapid increases in bacterial diversity up to approximately 3 years of age [17]. As such, it remains unclear if findings from paediatric studies extend to adult populations. Only a limited number of studies have examined the microbiota of adults with existing allergic disease. Of these few studies conducted, reduced total count of bacteria and anaerobic bacteria was reported in adult atopic dermatitis patients $(n=11)$ compared to the healthy controls $(n=14)$ [18]. This study employed bacterial culture techniques to count and identify specific bacterial taxa and its drawbacks including exclusion of "nonculturable" bacteria are well-recognised. 16s rRNA gene-based sequencing has been recently used to circumvent these restrictions, whereby a greater abundance of genera Faecalibacterium and Bifidobacterium is reported to exist in the faecal microbiota of adult allergic asthma sufferers $(n=21)$ com- pared to healthy individuals $(n=22)$ [8]. Similar microbial identification tools were used in the large-scale "American Gut Project," which comprised 1,879 participants. The results of this trial revealed a decreased microbial diversity in participants with self-reported drug, food, and seasonal allergies, a decreased abundance of Clostridiales, and a higher abundance of Bacteroidales in participants with self-reported seasonal allergies [10]. Given that allergy subtypes are mediated by different inflammatory pathways [19] and perhaps relate to different gut microbial profiles, there may be merit in classifying subjects by allergy phenotypes rather than self-reported allergen sensitivity.

To the authors' knowledge, no faecal microbiota studies employing next-generation sequencing technology have been conducted in adult AR sufferers. This study investigated this gap in knowledge by comparing the gut microbial composition between adults with well-defined $\mathrm{AR}$ and controls without $\mathrm{AR}$.

\section{Materials and Methods}

\section{Study Design}

This study was designed as a cross-sectional study to characterise differences in the gut microbiota between adults with established $\operatorname{AR}(n=57)$ and adults with no history of $\operatorname{AR}(n=23$; control group). All participants attended appointments at the Queensland Allergy Services Clinic in Southport (Gold Coast, Australia) and the Clinical Trial Unit at Griffith University (Gold Coast, Australia) for allergy testing and collection of blood and stool samples. This study was approved by the Griffith University Human Research Ethics Committee (Approval No. 2015/564/HREC; 2016/279).

\section{Participant Selection}

Men and women aged 18-65 years were recruited to the study. The age of participants was closely monitored throughout the recruitment process, and targeted age recruitment of participants was undertaken (when necessary) to ensure that the age profile of the AR and non-AR group was similar. Adults with established AR included both seasonal and perennial AR sufferers with a greater than 2-year history of AR symptoms and a positive allergic response to dust mites or grass pollens. Allergy status was confirmed with a skin prick test against a panel of dust mite and plant pollen allergens, as described previously $[20,21]$. AR participants were symptomatic at the time of sample collection and symptom severity was determined using the validated mini Rhinoconjunctivitis Quality of Life Questionnaire (mRQLQ) consisting of 14 questions separated into 5 domains: activities, practical problems, nose symptoms, eye symptoms, and other symptoms [22]. All items on the questionnaire were rated on a 7-point Likert scale (0-6), with each item averaged to give a maximum overall score of 6 .

AR subjects were excluded from participating if they suffered from non-AR (vasomotor rhinitis), consumed probiotics in the previous 8-12 weeks, were treated with oral corticosteroids within 
Table 1. Demographic and clinical features of AR participants and CG

\begin{tabular}{lcccc}
\hline & $\begin{array}{l}\text { All } \\
\text { mean }\end{array}$ & $\begin{array}{l}\text { AR } \\
\text { mean } \pm \text { SD }\end{array}$ & $\begin{array}{l}\text { CG } \\
\text { mean } \pm \text { SD }\end{array}$ & $p$ value \\
\hline$N$ & 80 & 57 & 23 & - \\
Age, years & $38.34 \pm 12.54$ & $39.06 \pm 13.29$ & $36.55 \pm 10.51$ & 0.421 \\
Sex, M/F & $32 / 48(60 \% \mathrm{~F})$ & $22 / 35(61 \% \mathrm{~F})$ & $10 / 13(57 \% \mathrm{~F})$ & 0.687 \\
Height, cm & $172.16 \pm 9.83$ & $171.07 \pm 9.80$ & $174.85 \pm 9.60$ & 0.120 \\
Weight, kg & $75.90 \pm 16.20$ & $76.27 \pm 14.95$ & $75.00 \pm 19.31$ & 0.779 \\
BMI, kg/m & $25.47 \pm 4.28$ & $25.96 \pm 4.08$ & $24.25 \pm 4.61$ & 0.105 \\
Ethnicity $\%$ Caucasian) & 87.50 & 84.21 & 95.65 & 0.161 \\
Immune measures & & & & 0.004 \\
$\quad$ WBC count $\left(\times 10^{9} / \mathrm{L}\right)$ & $6.48 \pm 1.69$ & $6.82 \pm 1.72$ & $5.63 \pm 1.30$ & 0.014 \\
Lymphocytes $\left(\times 10^{9} / \mathrm{L}\right)$ & $2.09 \pm 0.66$ & $2.21 \pm 0.66$ & $1.81 \pm 0.59$ & $<0.00001$ \\
Eosinophils $\left(\times 10^{9} / \mathrm{L}\right)$ & $0.30 \pm 0.26$ & $0.37 \pm 0.27$ & $0.11 \pm 0.08$ & 0.136 \\
Neutrophils $\left(\times 10^{9} / \mathrm{L}\right)$ & $3.52 \pm 1.16$ & $3.64 \pm 1.21$ & $3.22 \pm 0.98$ & 0.110 \\
Basophils $\left(\times 10^{9} / \mathrm{L}\right)$ & $0.05 \pm 0.04$ & $0.05 \pm 0.04$ & $0.04 \pm 0.03$ & 0.144 \\
ESR, mm/h & $9.10 \pm 9.83$ & $10.12 \pm 10.70$ & $6.57 \pm 6.81$ & - \\
Disease characteristics & & & & - \\
Co-allergy to dust mites and pollen, \% & 42.5 & 59.65 & 0 & - \\
Dust mite only, \% & 23.75 & 33.33 & 0 & 0 \\
Pollen only, \% & 5 & 7.02 & & \\
\hline
\end{tabular}

AR, allergic rhinitis; CG, control group; M, male; F, female; ESR, erythrocyte sedimentation rate.

the previous 6 months or antibiotics within the previous 30 days, used anti-inflammatory or immune-modulating medications, had existing respiratory disease including asthma, nasal polyposis or chronic obstructive pulmonary disorder, had existing immune dysfunction (other than allergies) or gastrointestinal tract diseases or disorders, were ill or had infectious disease at the time of enrolment, or were pregnant at the time of enrolment.

Individuals were recruited to the study as controls (CG) if they reported no history of $\mathrm{AR}$, tested negative to the panel of dust mite and plant pollens, and were free from chronic disease. Participants were excluded from the study if they consumed probiotics in the previous 8-12 weeks, had taken antibiotics within the previous 30 days, used anti-inflammatory or immune-modulating medications, had existing respiratory disease, immune dysfunction or gastrointestinal disease or disorder, were ill or had an infectious disease at the time of enrolment, or were pregnant at the time of enrolment.

\section{Blood Sample Collection}

Venous blood samples were collected for analysis of full blood count including white cell differential (QML Pathology, Murarrie, QLD, Australia). In addition, erythrocyte sedimentation rate (ESR) over $1 \mathrm{~h}$ was measured using fresh blood samples collected in sodium citrate tubes and using commercially available Vacuette ESR pipettes (Greiner Bio-One, Kremsmünster, Austria) as per the Westergren method [23].

\section{Stool Sample Collection}

Subjects were provided with a sample collection kit and instructed to collect a stool sample within $24 \mathrm{~h}$ prior to their scheduled study visit. Collection instructions included not to contami- nate the sample with urine or water and to store the sample at room temperature until their study visit. Stool samples were frozen at $-80^{\circ} \mathrm{C}$ upon receipt until processing.

\section{Faecal Microbial Composition}

DNA was extracted from defrosted stool samples using the method described by Yu et al. [24], which included homogenisation, a combination of chemical and mechanical lysis (using silica/ zirconia beads; Daintree Scientific, St Helens, TAS, Australia), salt/ alcohol precipitation, and purification using a Qiagen DNAeasy kit (Qiagen, Hilden, Germany). The quality and quantity of DNA were assessed with the NanoDrop $1000 \mathrm{UV}$-Vis spectrophotometer (Thermo Scientific, Waltham, MA, USA).

Isolated DNA was amplified using universal primers for the $\mathrm{V} 3-\mathrm{V} 4$ region of the microbial 16s rRNA gene (F: $5^{\prime}$-CCTACGGGNGGCWGCAG-3'; R: 5'GACTACHVGGGTATCTAATCC-3'), as described previously [25], and PCR products were sequenced on an Illumina MiSeq system (Illumina, CA, USA) by a commercial provider (Macrogen, Seoul, Korea). Sequence data were processed with CD-HIT-OTU [26] to filter out erroneous and chimaeric reads. Taxonomic classification and identity assignment was performed using a reference-based approach with the NCBI database of $16 \mathrm{~s}$ rRNA gene sequences.

\section{Statistics}

Differences in demographic and clinical measures between groups were assessed with an independent $t$ test for continuous variables and a $\chi^{2}$ test for categorical variables. A one-way ANOVA or an independent $t$ test was used to perform the differential a-diversity. Welch's $t$ test/unequal variances $t$ test was used for heteroscedastic data. A non-parametric Mann-Whitney $U$ test was 


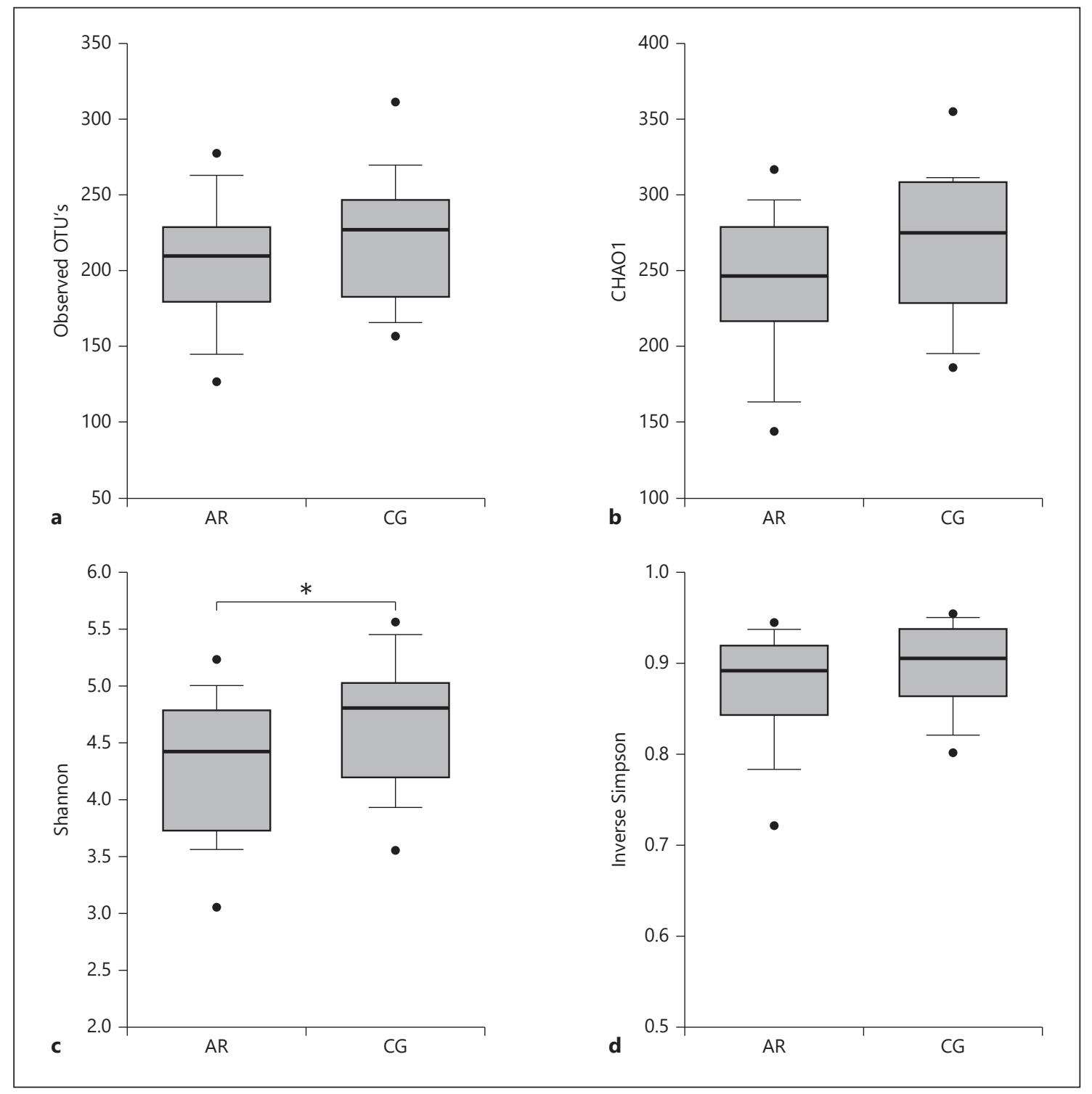

Fig. 1. a-d Alpha diversity metrics for allergic rhinitis (AR) and control group (CG). 16s rRNA sequencing was performed using stool samples collected from AR and CG participants and alpha diversity metrics were determined. a The total number of observed operational taxonomic units (OTUs) was lower in the AR group. b The CHAO1 index was lower in the AR group. c The Shannon

used to perform the abundance analyses from phylum to species levels. The analysis was confined to taxa with a relative abundance (detected) of $>0 \%$ and prevalence of $>50 \%$ in either group (AR or CG). Differences in detection rate of taxa (i.e., detected, $>0 \%$; or not detected, $0 \%$; in a given sample) between groups were identified with a $\chi^{2}$ test. Statistical significance was accepted at $p<0.05$. Partial least squares-discriminant analysis (PLS-DA) implemented in mixOmics R package [27] was employed for the multivariate analysis. Taxa with a relative abundance of $>0 \%$ and prevalence $<50 \%$ in both groups (AR and CG) were excluded from the PLS-DA.

Gut Microbiome of Adults with Allergic Rhinitis index was significantly lower in the AR group $\left({ }^{*} p<0.05\right)$. d The inverse Simpson index was lower in the AR group. Outer limits of each box represent the 25 th and 75 th percentiles, with the median shown as the line within the box. Whiskers (error bars) show the 5th and 95th percentiles, with filled circles showing the outliers.

\section{Results}

The demographic and clinical characteristics of the cohorts are included in Table 1 . The groups were matched in key physical attributes. However, consistent with diagnosis of atopic conditions, the AR group had significantly higher eosinophil counts than the control group (CG). The total WBC and lymphocyte counts were also signifi- 
cantly elevated in the AR group, although the average counts were within normal reference ranges. The AR group had mild-moderate symptoms based on mRQLQ scores $(2.84 \pm 1.23$ [out of a maximum possible score of 6]) with the majority sensitised to both plant pollens and dust mites. Several participants also reported allergies other than AR; a total of $44 \%$ of the cohort reported a history of skin allergies (eczema, hand dermatitis, urticaria, and itchy rash), $28 \%$ also reported a history of food allergy, and $12 \%$ also reported a history of drug allergy (including codeine [opioid], acetylsalicylic acid [non-steroidal anti-inflammatory drug], antibiotics, and metoclopramide [dopamine $\mathrm{D}_{2}$ receptor antagonist/5- $\mathrm{HT}_{3}$ receptor antagonist/5- $\mathrm{HT}_{4}$ receptor agonist]).

\section{Microbial Diversity}

A trend for greater microbial diversity $(\sim 7 \%$; $p=$ 0.129 ) was noted among the CG. Higher operational taxonomic unit (OTU) count, CHAO1, and inverse Simpson a-diversity measures were observed in the CG compared to the AR group (Fig. 1). The Shannon diversity index, which considers both species richness and evenness, was significantly higher $(p=0.037)$ in the controls $(C G)$ than in the AR group (Fig. 1).

\section{Microbial Composition/Taxonomic Classification Phyla}

Taxonomic classification at the phylum level revealed that Firmicutes, Bacteroidetes, Verrucomicrobia, and Proteobacteria were the dominant bacterial phyla in both groups (Fig. 2). The Bacteroidetes (34.36\% [27.6743.21\%] in AR vs. $28.74 \%$ [22.35-35.78\%] in CG, U 425, $p=0.014)$ was significantly more abundant in the AR group than in the CG. In contrast, the Firmicutes phylum was significantly less abundant in the AR group than in the CG (57.24\% [47.29-64.77\%] vs. 64.44\% [57.60$71.46 \%$ ], U 396, $p=0.006)$. Further, there was a significant difference in the Firmicutes to Bacteroidetes ratio between the AR group and CG (1.73\% [1.11-2.08\%] vs. $2.33 \%$ [1.76-3.05\%], U 377, $p=0.003)$.

\section{Order}

Within the order taxonomic rank, the OTUs obtained were assigned to 26 unique orders; of these, 12 orders were considered prevalent (detected in $>50 \%$ of samples from either group) and 14 were less prevalent detected in $<50 \%$ of samples), see online suppl. Table 1 ; see www. karger.com/doi/10.1159/000510536 for all online suppl. material. The most abundant order present in the both AR and CG cohorts were Clostridiales (53.14\%), Bacte-

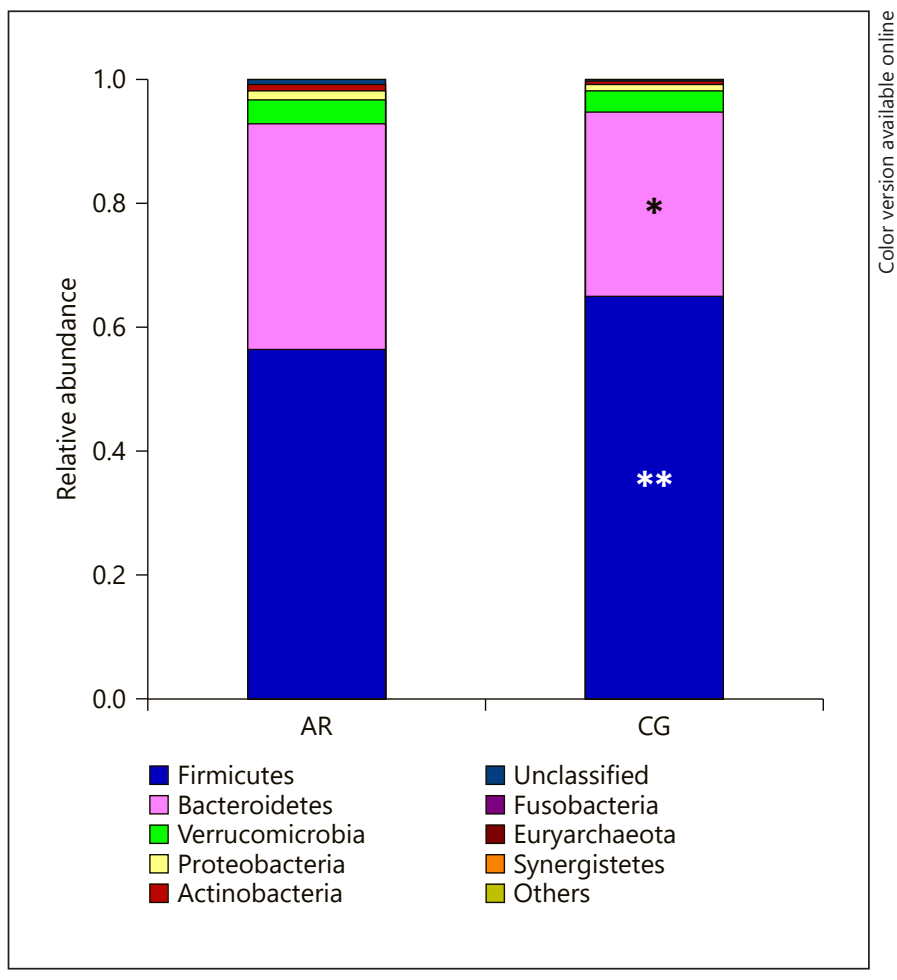

Fig. 2. The relative abundance of bacteria phyla for allergic rhinitis (AR) group and control group (CG) measured via $16 \mathrm{~s}$ rRNA sequencing using collected stool samples. ${ }^{*} p<0.05,{ }^{* *} p<0.01$.

roidales (34.13\%), Negativicutes unclassified (4.32\%), and Verrucomicrobiales (3.76\%). The order Bacteroidales was significantly more abundant in the AR cohort than in the CG cohort. In contrast, the Clostridiales was significantly less abundant in the AR cohort than in the CG cohort (Table 2).

\section{Genus}

At the genus level, 112 unique genera were identified; 57 of these genera were considered prevalent and 55 were less prevalent (online suppl. Table 2). The most abundant genera in both the AR and CG were Bacteroides (21.55\%), Faecalibacterium (20.03\%), Lachnospiraceae unclassified genera (6.25\%), Alistipes (4.15\%), Akkermansia (3.77\%), Prevotella (3.71\%), and Oscillibacter (3.51\%). The genus Parabacteroides was significantly more abundant in the AR group than in the CG (Table 2). In contrast, Coprococcus, Oxalobacter, and Sutterella were significantly less abundant in the AR group (Table 2).

Among the less-prevalent genera, some differences in rates of detection between AR and CG were noted (Table 3). For example, the Acidaminococcus genus was de-
Watts/West/Zhang/Smith/Cripps/Cox 
Table 2. Relative abundance (\%) of differentially abundant taxa between the AR and CG at the order, genus and species level

\begin{tabular}{|c|c|c|c|c|c|c|}
\hline & \multicolumn{2}{|l|}{ Mean and SD } & \multicolumn{2}{|c|}{ Median and 25-75th percentile } & $U$ statistic & $p$ value \\
\hline Bacteroidales & $36.30 \pm 12.10$ & $28.70 \pm 7.97$ & $34.36(27.67-43.19)$ & $27.68(22.27-33.74)$ & 385 & 0.004 \\
\hline Clostridiales & $50.50 \pm 14.70$ & $59.80 \pm 10.80$ & $53.36(40.59-59.33)$ & $60.85(55.22-67.28)$ & 390 & 0.005 \\
\hline \multicolumn{7}{|l|}{ Genus } \\
\hline Oxalobacter & $<0.01 \pm<0.01$ & $<0.01 \pm 0.01$ & $<0.01(<0.01-<0.01)$ & $<0.01(<0.01-0.01)$ & 401 & 0.001 \\
\hline Sutterella & $0.07 \pm 0.13$ & $0.14 \pm 0.22$ & $<0.01(<0.01-0.10)$ & $0.08(0.01-0.18)$ & 413 & 0.007 \\
\hline Faecalibacterium & $19.30 \pm 12.10$ & $21.80 \pm 11.50$ & $18.51(10-27.65)$ & $25.14(8.74-32.16)$ & 574 & 0.386 \\
\hline \multicolumn{7}{|l|}{ Species } \\
\hline Bacteroides massiliensis & $0.66 \pm 1.19$ & $1.24 \pm 1.72$ & $<0.00(0.00-1.06)$ & $0.77(<0.00-2.01)$ & 464 & 0.029 \\
\hline Intestinimonas butyriciproducens & $0.24 \pm 0.42$ & $0.66 \pm 1.15$ & $0.06(0.02-0.23)$ & $0.26(0.05-0.67)$ & 406.5 & 0.008 \\
\hline Oscillibacter valericigenes & $0.23 \pm 0.33$ & $0.45 \pm 0.47$ & $0.12(0.02-0.24)$ & $0.25(0.16-0.65)$ & 408 & 0.008 \\
\hline Agathobaculum butyriciproducens & $0.12 \pm 0.09$ & $0.24 \pm 0.24$ & $0.09(0.05-0.16)$ & $0.15(0.08-0.30)$ & 433 & 0.018 \\
\hline Anaerotruncus colihominis & $0.03 \pm 0.03$ & $0.02 \pm 0.01$ & $0.02(0.01-0.05)$ & $0.01(<0.01-0.03)$ & 418 & 0.012 \\
\hline Oxalobacter formigenes & $<0.01 \pm 0.01$ & $0.01 \pm 0.01$ & $<0.01(<0.01-<0.01)$ & $<0.01(<0.01-0.01)$ & 400 & 0.001 \\
\hline Sutterella wadsworthensis & $0.05 \pm 0.11$ & $0.09 \pm 0.19$ & $<0.01(<0.01-0.03)$ & $0.02(<0.01-0.09)$ & 473 & 0.037 \\
\hline
\end{tabular}

AR, allergic rhinitis; $C G$, control group.

tected significantly more frequently in the AR group than in the CG ( $25 \%$ of AR samples vs. $\sim 4 \%$ of non-AR samples). In contrast, the genera Rothia and Coriobacteriaceae unclassified were detected significantly more often in the CG than in the AR group.

Species

At the species level, 290 unique species were identified - 122 of these species were considered prevalent and 168 were less prevalent. Anaerotruncus colihominis was significantly more abundant in the AR group than in the CG (Table 2). In contrast, Bacteroides massiliensis, Intestinimonas butyriciproducens, Eubacterium xylanophilum, Murimonas intestini, Clostridium asparagiforme, Coprococcus eutactus, Oscillibacter valericigenes, Agathobaculum butyriciproducens, Oxalobacter formigenes, and Sutterella wadsworthensis were less abundant in the AR group than in the CG (Table 2).

Differences in the detection rate of less-prevalent species $(<50 \%$ of either cohort) were observed between the $\mathrm{AR}$ and CG cohort (Table 3). Clostridium hylemonae, $R u$ minococcus gnavus, and Acidaminococcus intestini species were present significantly more in the AR cohort than in the CG. In contrast, Rothia mucilaginosa, Muricomes intestini, Clostridium papyrosolvens, Clostridium straminisolvens, and Dialister succinatiphilus were detected significantly less frequently in the AR cohort than in the CG.

\section{PLS-DA Multivariate Analysis}

Multivariate analysis using PLS-DA was performed on phylum to species level with microbiota abundance data. As shown in Figure 3a and b, PLS-DA discriminated the $A R$ and CG cohorts based on the genus and species abundance data. Clustering of samples was observed at the genus and species levels for the first 2 principal coordinates, suggesting a distinct microbial structure between cohorts, although the observed clustering of samples accounts for a small amount of total variance.

\section{Discussion}

The current study analysed the faecal microbial community of adult AR sufferers and controls. The AR cohort had a distinct gut microbiome profile, marked by a re- 
Table 3. Differentially detected taxa between the AR and CG at the family, genus, and species level

\begin{tabular}{|c|c|c|c|c|}
\hline & $\begin{array}{l}\mathrm{AR} \\
n(\%)\end{array}$ & $\begin{array}{l}\text { CG } \\
n(\%)\end{array}$ & $\begin{array}{l}\text { Fisher's exact } \\
p \text { value }\end{array}$ & $\begin{array}{l}\chi^{2} \\
p \text { value }\end{array}$ \\
\hline \multicolumn{5}{|l|}{ Family } \\
\hline Micrococcaceae & $4(7.02)$ & $7(30.43)$ & 0.003 & 0.0001 \\
\hline Oxalobacteraceae & $10(17.54)$ & $13(56.52)$ & 0.0009 & 0.0005 \\
\hline \multicolumn{5}{|l|}{ Genus } \\
\hline Rothia & $4(7.02)$ & $7(30.43)$ & 0.003 & 0.0001 \\
\hline Coriobacteriaceae unclassified & $7(12.28)$ & $10(43.48)$ & 0.0049 & 0.0020 \\
\hline Acidaminococcus & $14(24.56)$ & $1(4.35)$ & 0.0548 & 0.0360 \\
\hline Oxalobacter & $10(17.54)$ & $13(56.52)$ & 0.0009 & 0.0005 \\
\hline Sutterella & $25(43.86)$ & $19(82.61)$ & 0.0025 & 0.0016 \\
\hline \multicolumn{5}{|l|}{ Species } \\
\hline Rothia mucilaginosa & $4(7.02)$ & $7(30.43)$ & 0.0147 & 0.0092 \\
\hline Bacteroides massiliensis & $23(40.35)$ & $16(69.57)$ & 0.0258 & 0.0180 \\
\hline Christensenella minuta & $45(78.95)$ & $23(100)$ & 0.0154 & 0.0170 \\
\hline Muricomes intestini & $0(0)$ & $2(8.70)$ & 0.0801 & 0.0242 \\
\hline Murimonas intestini & $31(54.39)$ & $19(82.61)$ & 0.223 & 0.0183 \\
\hline Clostridium asparagiforme & $23(40.35)$ & $15(65.22)$ & 0.0517 & 0.0438 \\
\hline Clostridium hylemonae & $27(47.37)$ & $4(17.39)$ & 0.0212 & 0.0127 \\
\hline Ruminococcus gnavus & $26(45.61)$ & $5(21.74)$ & 0.0748 & 0.0473 \\
\hline Clostridium papyrosolvens & $5(8.77)$ & $6(26.09)$ & 0.0687 & 0.0418 \\
\hline Clostridium straminisolvens & $4(7.02)$ & $8(34.78)$ & 0.0036 & 0.0016 \\
\hline Acidaminococcus intestini & $13(22.81)$ & $1(4.35)$ & 0.0568 & 0.0492 \\
\hline Dialister succinatiphilus & $9(15.79)$ & $9(39.13)$ & 0.0371 & 0.0237 \\
\hline Oxalobacter formigenes & $10(17.54)$ & $13(56.52)$ & 0.0009 & 0.0005 \\
\hline Sutterella wadsworthensis & $23(40.35)$ & $16(69.57)$ & 0.0258 & 0.0180 \\
\hline
\end{tabular}

In a given sample, taxa were considered "detected" if the relative abundance was $>0 \%$ and "not detected" if the relative abundance was $0 \%$. AR, allergic rhinitis; CG, control group.

duced microbial diversity and altered abundance of certain gut microbes compared to the controls. The results presented here provide evidence that unique gut microbial patterns occur in adult AR sufferers and warrant further investigation in the form of mechanistic studies.

A key finding from the current study was related to overall microbial diversity. Species richness ( $\alpha$-diversity) measured via the Shannon index was significantly reduced in the AR cohort compared to the CG. A similar trend of reduced species richness in the AR cohort was also observed with the inverse Simpson, observed OTU counts, and CHAO1 diversity indices. Lack of sufficient richness or evenness in the gastrointestinal microbial community appears to impair its ability to withstand exogenous disturbances [28]. Indeed, it has been suggested that reliable microbial richness indices may be useful indicators to determine the relative stability or "fitness" of the gut microbiome [28]. Other studies have also identified reduced richness in atopic individuals compared to controls. Hua et al. [10] examined publicly available $16 \mathrm{~S}$
rRNA data collected from the American Gut Project and reported that species richness was significantly negatively associated with self-reported seasonal allergy in adult sufferers. Similarly, Bisgaard et al. [29] reported that reduced faecal bacterial diversity at 1 and 12 months after birth significantly increased the risk of developing allergic sensitisation and AR by the age of 6 years $(n=346)$.

Other key findings from the current study relate to the differential abundance/detection of particular microbial taxa or specific microbes in the AR group compared to the controls. At the phylum level, a significantly different Firmicutes to Bacteroidetes ratio between groups, with a higher abundance of Bacteroidetes and lower abundance of Firmicutes in the AR cohort were detected. Increased abundance of Bacteroidetes in the AR cohort translated to the class (Bacteroidia) and order (Bacteroidales) taxa. Other studies of atopic cohorts have also identified a higher abundance of Bacteroidetes classifications in stool samples from atopic subjects. Analysis of data from the American Gut Project identi- 


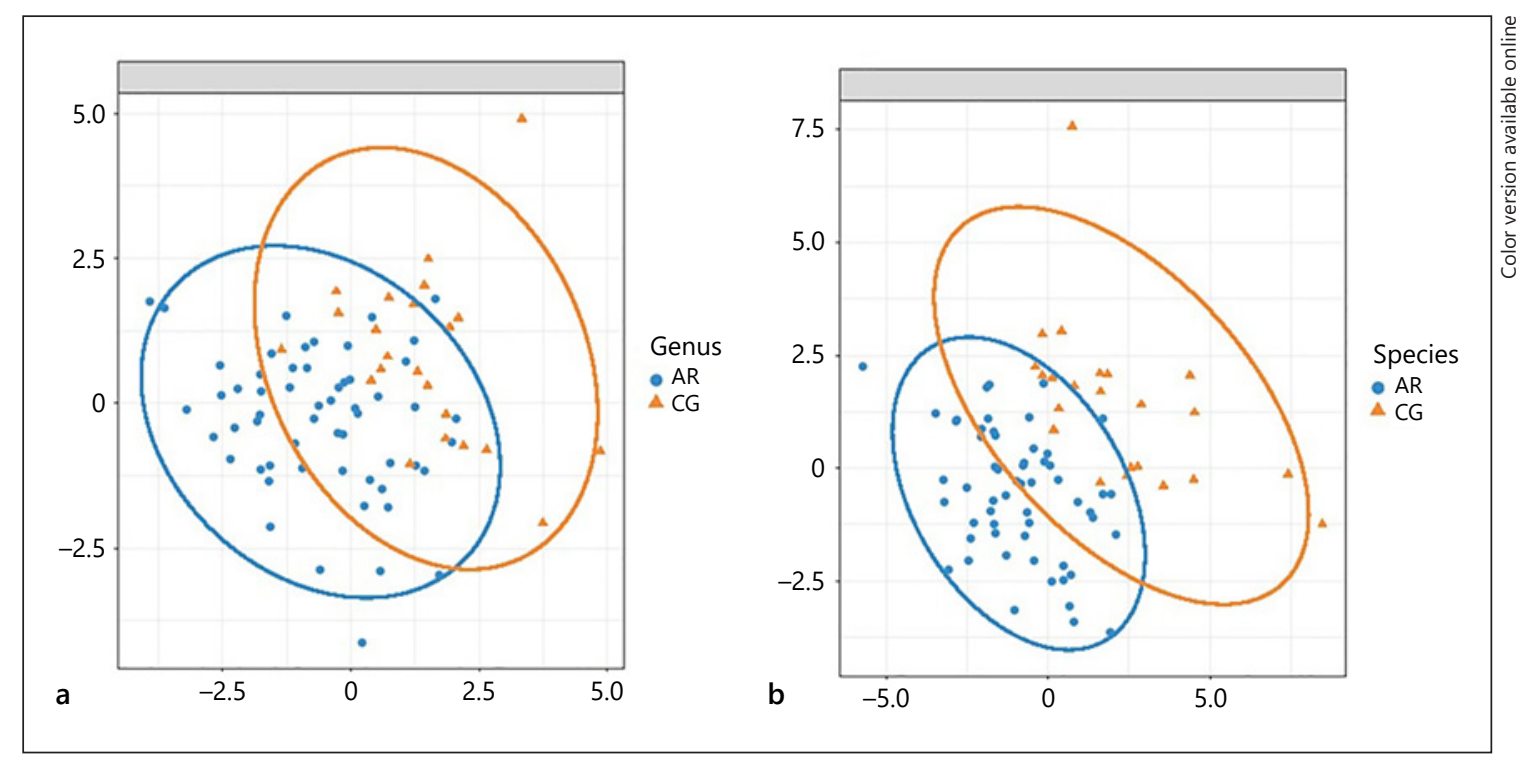

Fig. 3. Multivariate analysis using partial least squares-discriminant analysis at the genus and species level collated from 16s rRNA sequencing data performed using collected stool samples. a At the genus level, clustering of samples within the AR and CG cohorts was observed for the first 2 principal coordinates. The first coordinate represents $7 \%$ variance explained and second coordinate represents $6 \%$ variance explained. b At the species level, clear cluster- ing of allergic rhinitis (AR) and control group (CG) samples was also observed for the first 2 principal coordinates. The first coordinate represents $7 \%$ of variance explained and the second coordinate represents $4 \%$ of variance explained. The blue full circles represent the AR samples and the orange full triangles represent the CG samples. The coloured ellipses, blue for AR and orange for CG, are plotted to represent the $95 \%$ confidence level of the population. fied a higher abundance of Bacteroidales in adults with self-reported nut and seasonal pollen allergies [10]. In a small study of Japanese infants $(n=15)$, the abundance of Bacteroidaceae was significantly higher in infants who later went on to develop atopic disease by the age of 2, as determined by the International Study of Asthma and Allergies in Childhood (ISAAC) questionnaire [30]. It is worth noting that Bacteroidetes and Firmicutes are the most abundant phyla present in the western faecal microbiome $[31,32]$, and this observation was also reflected in this study. The metabolites generated by these phyla play a significant role in colonic health and immune regulation. The Bacteroidetes phyla are generally associated with a greater production of acetate and propionate, whereas the Firmicutes phyla are associated with a greater production of butyrate [33]. Butyrate is a key energy source for colonic epithelial cells and contributes to maintaining the intestinal barrier via modulation of tight junction expression [34]. A dysfunctional gut barrier allows for increased pro-inflammatory molecules and antigen transfer into submucosa and systemic circulation, resulting in local and systemic inflammatory responses. A dominance of
Bacteroidetes over Firmicutes may reduce overall butyrate production and thereby affect the integrity of the gut barrier. Interestingly, clinical and experimental studies have shown that gut permeability is increased in subjects with allergic disease compared to healthy controls [35-40]. Given that these results were generated from independent studies, future work is needed to explore the potential link between abundance of butyrateproducing bacteria and gut permeability in atopy.

At the class and order level, this study reports a reduced abundance of Clostridia/Clostridiales (Firmicutes) in the AR cohort compared to the controls. A reduced abundance of Clostridiales was also observed by Hua et al. [10] in adults with self-reported nut and seasonal pollen allergies. Reduced Clostridia has also been detected in faecal samples of atopic infants and children. Candela et al. [41] reported a lower count of Clostridium $I V$ in their cohort of Italian children ( $n=19$ allergic, 12 non-allergic; aged 4-14 years). Verhulst et al. [42] observed an association between reduced prevalence of Clostridium in stool samples of infants $(n=154)$ at 3 weeks of age and the occurrence of wheezing symptoms at 12 months of age. Indigenous intestinal Clostridium clusters IV, XIVa, and 
XVIII have been recognised as effective inducers of Tregs in the colon of mice $[43,44]$. Tregs are known for their ability to maintain immune homeostasis and promote immune tolerance to allergens, which is particularly relevant in the pathogenesis of allergic diseases [45]. Notably, reduced Clostridia has also been identified in other immune-mediated diseases such as Crohn's disease [46].

At the genus level, we observed a higher abundance of Parabacteroides (Bacteroidetes) and a reduced abundance of Coprococcus (Firmicutes), Sutterella (Proteobacteria), and Oxalobacter (Proteobacteria) in the AR group compared to the CG. No direct associations between the faecal abundance of the butyrate-producing Coprococcus species and adults with AR have been previously reported. However, Simonyté Sjödin et al. [47] reported an underrepresentation of Coprococcus abundance in stool samples from children with a history of allergic disease (21 allergic, 72 non-allergic) from infancy to 8 years of age. While we emphasise the link between this bacterial genus and $\mathrm{AR}$, a reduced abundance of Coprococcus has also been reported in adults with other inflammatory conditions such as ESRD [48] and psoriatic arthritis [49]. The current study is the first to report a link between AR and Sutterella abundance in adult stool samples. The Sutterella species have been reported to be highly prevalent in the gut mucosa of humans possibly due to their ability to resist bile acids [50]. A reduced Sutterella abundance has also been reported in other atopic conditions such as IgE-associated eczema in infants $(n=20)$ compared with non-atopic infants $(n=20)$ at 1 and 12 months of age. A further interesting and novel finding in the present study was the reduced abundance of the genus Oxalobacter (Proteobacteria) in the AR cohort. Members of the Oxalobacteraceae are known to colonise the rhizosphere and roots of many plant species [51]. In relation to human health, Oxalobacter species metabolise oxalate in the intestinal tract and is protective against the formation of calcium oxalate kidney stones and other oxalate-associated pathologies [52]. Furthermore, a link between the presence of Oxalobacteraceae in house dust and the prevalence of atopy has been observed. Indeed, members of the Oxalobacteraceae were found to be more abundant in dust samples from the Finnish Karelia homes compared to geographically adjacent Russian Karelia, whereby the abundance of atopic disease in this region is 4-fold lower [53]. While microbial gut composition was not performed in allergy sufferers living in these regions, these findings provide a potential link between exposure to plant-related microbes such as Oxalobacteraceae and the prevalence of atopy.
In the current study, numerous species were differentially abundant/detected (11 abundant/14 detected) in the AR group compared to the controls. Among these species, several were identified as previously associated with atopy; however, for a large proportion of the differentially abundant/detected species, the relationship with atopy is unknown and warrants further investigation. Species differentially abundant/detected and of particular interest include S. wadsworthensis (Proteobacteria), C. eutactus (Firmicutes), and R. gnavus (Firmicutes). The finding of a reduced abundance of $S$. wadsworthensis and C. eutactus in the AR group is consistent with a previous report in an atopic cohort. In a study of adults residing in the UK with asthma ( $n=36$ asthma, 185 controls), a reduced enrichment of $S$. wadsworthensis and C. eutactus was observed in the asthma group compared with the controls [54]. Interestingly, C. eutactus produces the short chain fatty acid butyrate, which is a known inducer of colonic regulatory $T$ cells [55]. The authors suggest that the depletion of butyrate-producing bacteria, such as $C$. eutactus, may be linked with the presentation of asthma. In the current study, a significantly increased detection of $R$. gnavus in the AR group when compared to the CG was observed. R. gnavus has been previously associated with the development and pathogenesis of atopy, especially respiratory allergies [56]. Chua et al. [56] reported a higher incidence of $R$. gnavus in stool specimens from infants who later developed respiratory allergies. In a follow-up experimental asthmatic mouse model, ovalbumin-sensitised/challenged mice were infected with $R$. gnavus via oral gavage intragastric administration. The R. gnavusinfected mice showed greater secretion of interleukin (IL)-25, IL-33, and thymic stromal lymphopoietin by colonic tissues, thereby promoting Th2 differentiation and further cytokine release, and an enhanced infiltration of eosinophils and mast cells to the colon and lung parenchyma [56]. In addition, the R. gnavus-infected mice displayed increased airway hyperresponsiveness and histologic airway inflammation [56], providing evidence of a clear link between gut bacterial species and mechanisms underpinning allergic disease.

Key differentially abundant species identified in previous reports of atopic children and infants, including Akkermansia muciniphila, Faecalibacterium prausnitzii, Bifidobacterium catenulatum, Bifidobacterium longum, Staphylococcus aureus, Bacteroides fragilis, Clostridium difficile, Bacteroides Vulgatus, and Escherichia coli, were not significantly differentially abundant in this cohort of adults suffering AR. This finding may be due to differences in study features, cohort ethnicity, 
sample processing methodology, and microbial identification tools used. Nonetheless, the differentially abundant microbes present in infancy/childhood cannot be entirely extrapolated to adults and therefore demonstrates the importance of sampling the microbiome of allergy sufferers in adulthood. Longitudinal studies that capture the early microbiome and the microbiome throughout childhood to adulthood, while a challenging task, are worth further investigation to elucidate shifts in the microbiome of allergic subjects over time. In addition, mechanistic studies in an animal model such as gnotobiotic mice could be conducted to elucidate how the taxa identified in the current study contribute to the pathophysiology of AR.

The strengths of the current study lie in the novelty of assessing gut microbiome composition in an adult population with clinically well-characterised allergic disease. In addition, notwithstanding population studies, the sample size in this investigation is larger than typical single-centre investigations. Despite the strength of the design, the authors acknowledge that this study is not without its limitations. These particularly relate to the use and interpretation of $16 \mathrm{~s}$ rRNA amplicon analysis in providing high resolution of the microbial population at the species level. Nevertheless, 16s rRNA sequencing is often employed in human microbiome studies due to its ability to resolve the microbial population structure and biodiversity and its relative affordability. Additionally, this study describes the microbial composition of stool samples from adults with AR and adults without AR. Analysis of stool samples has the limitation of capturing luminal microbiota and not the mucosal-associated microbiota, which may play a critical role in regulation of the mucosal immune system and local mucosal immune regulation relevant in AR. However, without invasive procedures, there are no real alternatives. Cohort factors such as lifestyle, diet, and age were also not evaluated in this report, and therefore, the effect of these variables on the gut microbiome could not be quantified as this study was not sufficiently powered to conduct these analyses. However, a post hoc analysis to examine the impact of co-existing skin allergies on the gut microbiome was undertaken. Approximately $11 \%(6 / 57)$ of the AR group reported a personal history of urticaria and $12 \%(7 / 57)$ reported a personal history of eczema. Removing participants in the AR group with either a personal history of urticaria or eczema did not change the observations at the phyla, order, and genus level. In addition, post hoc correlation analyses of the AR group were performed with Spearman's test to examine the effect of mRQLQ scores on the gut microbi- ome. No significant correlation was observed between the mRQLQ scores and diversity indices or relative abundance of taxa at the phyla, order, genus, and species. To prevent the confounding effect of antibiotic exposure on the gut microbiome, participants who had taken antibiotics in the 30 days prior to enrolment were excluded from participation. However, it should be noted that the effect of antibiotic usage prior to this period ( $>30$ days prior to enrolment) was not evaluated in this study. The relationship between the immune parameters and the gut microbiome composition was not explored in this study. Mechanistic studies are needed to better understand the effect of specific gut microbial taxa and microbiota population structures on key effector cells and mediators involved in the allergic response.

Overall, a unique microbial community in the AR cohort, marked by a reduced microbial diversity, increased abundance of Bacteroidetes and Parabacteroides, and a reduced abundance of Oxalobacter, Sutterella, Coprococcus, and Clostridiales was observed in the current study. Several taxa identified in our study were consistent with previous reports in atopic adults. However, this study also identified taxa that were unique to our study and have not been previously associated with atopy. Interestingly, the differentially abundant/detected taxa reported here were not always consistent with the findings presented in atopic paediatric cohorts. In light of the unique microbiome patterns in adult AR subjects presented here, identifying the metabolites and mechanisms underpinning the microbiota-host relationship will improve the understanding of how the composition of the microbiome regulates immune homeostasis and may advise potential therapeutic options for treating allergies (e.g., dietary intervention, probiotics, and faecal transplant).

\section{Acknowledgements}

The authors appreciate the support of the Queensland Allergy Services Clinic for providing clinic access and for performing the skin prick tests.

\section{Statement of Ethics}

This study was approved by the Griffith University Human Research Ethics Committee (Approval Nos. 2015/564/HREC; 2016/279). All subjects provided written informed consent prior to participation. 


\section{Conflict of Interest Statement}

The authors report no conflicts of interest in relation to this work.

\section{Author Contributions}

A.M.W., N.P.W., and A.J.C. designed the study. A.M.W. performed the experiments and data analysis. P.Z. performed the PLS-DA statistical analysis. A.M.W. and A.J.C. drafted the manuscript. A.W.C., N.P.W., P.Z., and P.K.S. revised the manuscript. All authors approved the final version of the paper.

\section{References}

1 Alkanani AK, Hara N, Gottlieb PA, Ir D, Robertson $\mathrm{CE}$, Wagner $\mathrm{BD}$, et al. Alterations in intestinal microbiota correlate with susceptibility to type 1 diabetes. Diabetes. 2015; 64(10):3510-20.

2 Murri M, Leiva I, Gomez-Zumaquero JM, Tinahones FJ, Cardona F, Soriguer F, et al. Gut microbiota in children with type 1 diabetes differs from that in healthy children: a casecontrol study. BMC Med. 2013;11(1):46.

3 Scher JU, Sczesnak A, Longman RS, Segata N, Ubeda C, Bielski C, et al. Expansion of intestinal Prevotella copri correlates with enhanced susceptibility to arthritis. Elife. 2013; 2:e01202.

4 Vaahtovuo J, Munukka E, Korkeamäki M, Luukkainen R, Toivanen P. Fecal microbiota in early rheumatoid arthritis. J Rheumatol. 2008;35(8):1500-5.

5 Joossens M, Huys G, Cnockaert M, De Preter V, Verbeke K, Rutgeerts P, et al. Dysbiosis of the faecal microbiota in patients with Crohn's disease and their unaffected relatives. Gut. 2011;60(5):631-7.

6 Sokol H, Seksik P, Furet JP, Firmesse O, NionLarmurier I, Beaugerie L, et al. Low counts of Faecalibacterium prausnitzii in colitis microbiota. Inflamm Bowel Dis. 2009;15(8):11839.

7 Chen J, Chia N, Kalari KR, Yao JZ, Novotna M, Paz Soldan MM, et al. Multiple sclerosis patients have a distinct gut microbiota compared to healthy controls. Sci Rep. 2016;6: 28484.

8 Hevia A, Milani C, López P, Donado CD, Cuervo A, González S, et al. Allergic patients with long-term asthma display low levels of Bifidobacterium adolescentis. PLoS One. 2016;11(2):e0147809.

9 Watanabe S, Narisawa Y, Arase S, Okamatsu $\mathrm{H}$, Ikenaga T, Tajiri Y, et al. Differences in fecal microflora between patients with atopic dermatitis and healthy control subjects. J Allergy Clin Immunol. 2003;111(3):587-91.

10 Hua X, Goedert JJ, Pu A, Yu G, Shi J. Allergy associations with the adult fecal microbiota: analysis of the American Gut Project. EBioMedicine. 2016;3:172-9.

11 Melli LC, do Carmo-Rodrigues MS, AraújoFilho HB, Solé D, de Morais MB. Intestinal microbiota and allergic diseases: a systematic review. Allergol Immunopathol. 2016;44(2): $177-88$.
12 Prescott SL, Macaubas C, Holt BJ, Smallacombe TB, Loh R, Sly PD, et al. Transplacental priming of the human immune system to environmental allergens: universal skewing of initial T cell responses toward the Th2 cytokine profile. J Immunol. 1998;160(10):47307.

13 Wegmann TG, Lin H, Guilbert L, Mosmann TR. Bidirectional cytokine interactions in the maternal-fetal relationship: is successful pregnancy a $\mathrm{TH} 2$ phenomenon? Immunol Today. 1993;14(7):353-6.

14 McLoughlin RM, Mills KH. Influence of gastrointestinal commensal bacteria on the immune responses that mediate allergy and asthma. J Allergy Clin Immunol. 2011;127(5): 1097-9; quiz 108-9.

15 Mazmanian SK, Liu CH, Tzianabos AO, Kasper DL. An immunomodulatory molecule of symbiotic bacteria directs maturation of the host immune system. Cell. 2005;122(1): 107-18.

16 Ohnmacht C, Park JH, Cording S, Wing JB, Atarashi K, Obata Y, et al. Mucosal immunology. The microbiota regulates type $2 \mathrm{immu-}$ nity through RORgammat(+) T cells. Science. 2015;349(6251):989-93.

17 Yatsunenko T, Rey FE, Manary MJ, Trehan I, Dominguez-Bello MG, Contreras M, et al. Human gut microbiome viewed across age and geography. Nature. 2012;486(7402):222-7.

18 Matsumoto MM, Ohishi HH, Kakizoe KK, Benno YY. Faecal microbiota and secretory immunoglobulin a levels in adult patients with atopic dermatitis. Microb Ecol Health Dis. 2004;16(1):13-7.

19 Agache I, Rogozea L. Endotypes in allergic diseases. Curr Opin Allergy Clin Immunol. 2018;18(3):177-83

20 Watts AM, West NP, Smith PK, Cripps AW, Cox AJ. Probiotics and allergic rhinitis: a Simon two-stage design to determine effectiveness. J Altern Complement Med. 2016;22(12): 1007-12.

21 Watts AM, Cox AJ, Smith PK, Besseling-van der Vaart I, Cripps AW, West NP. A specifically designed multispecies probiotic supplement relieves seasonal allergic rhinitis symptoms. J Altern Complement Med. 2018;24(8): $833-40$.

22 Juniper EF, Thompson AK, Ferrie P, Roberts JN. Development and validation of the mini rhinoconjunctivitis quality of life questionnaire. Clin Exp Allergy. 2000;30(1):132-40.
23 Von Boroviczeny LEB KG, Bull BS, Chattas A, Dawson JB, Fukutake K, Gunz FW, et al. Recommendation of measurement of erythrocyte sedimentation rate of human blood. Am J Clin Pathol. 1977;68(4):505-7.

$24 \mathrm{Yu} \mathrm{Z}$, Morrison M. Improved extraction of PCR-quality community DNA from digest and fecal samples. Biotechniques. 2004;36(5): 808-12.

25 Klindworth A, Pruesse E, Schweer T, Peplies J, Quast C, Horn M, et al. Evaluation of general 16S ribosomal RNA gene PCR primers for classical and next-generation sequencingbased diversity studies. Nucleic Acids Res. 2013;41(1):e1.

26 Li W, Fu L, Niu B, Wu S, Wooley J. Ultrafast clustering algorithms for metagenomic sequence analysis. Brief Bioinformatics. 2012; 13(6):656-68.

27 Le Cao K, Rohart F, Gonzalez I, Dejean S, Gautier B, Bartolo F, et al. mixOmics: Omics Data Integration Project. R package version 6.1.1. 2016. Available from: https://CRANRprojectorg $/$ package $=$ mixOmics .

28 Bäckhed F, Fraser CM, Ringel Y, Sanders ME, Sartor RB, Sherman PM, et al. Defining a healthy human gut microbiome: current concepts, future directions, and clinical applications. Cell Host Microbe. 2012;12(5):611-22.

29 Bisgaard H, Li N, Bonnelykke K, Chawes BLK, Skov T, Paludan-Müller G, et al. Reduced diversity of the intestinal microbiota during infancy is associated with increased risk of allergic disease at school age. J Allergy Clin Immunol. 2011;128(3):646-52.e1-5.

30 Songjinda P, Nakayama J, Tateyama A, Tanaka S, Tsubouchi M, Kiyohara C, et al. Differences in developing intestinal microbiota between allergic and non-allergic infants: a pilot study in Japan. Biosci Biotechnol Biochem. 2007;71(9):2338-42.

31 The Human Microbiome Project Consortium. Structure, function and diversity of the healthy human microbiome. Nature. 2012; 486(7402):207-14

32 Johnson EL, Heaver SL, Walters WA, Ley RE. Microbiome and metabolic disease: revisiting the bacterial phylum Bacteroidetes. J Mol Med. 2017;95(1):1-8.

33 Den Besten G, van Eunen K, Groen AK, Venema K, Reijngoud DJ, Bakker BM. The role of short-chain fatty acids in the interplay between diet, gut microbiota, and host energy metabolism. J Lipid Res. 2013;54(9):2325-40. 
34 Brahe LK, Astrup A, Larsen LH. Is butyrate the link between diet, intestinal microbiota and obesity-related metabolic diseases? Obes Rev. 2013;14(12):950-9.

35 Ukabam SO, Mann RJ, Cooper BT. Small intestinal permeability to sugars in patients with atopic eczema. Br J Dermatol. 1984;110(6): $649-52$.

36 Pike MG, Heddle RJ, Boulton P, Turner MW, Atherton DJ. Increased intestinal permeability in atopic eczema. J Invest Dermatol. 1986; 86(2):101-4.

37 Rosenfeldt V, Benfeldt E, Valerius NH, Paerregaard A, Michaelsen KF. Effect of probiotics on gastrointestinal symptoms and small intestinal permeability in children with atopic dermatitis. J Pediatr. 2004;145(5):612-6.

38 Hijazi Z, Molla AM, Al-Habashi H, Muawad WM, Molla AM, Sharma PN. Intestinal permeability is increased in bronchial asthma. Arch Dis Child. 2004;89(3):227-9.

39 Benard A, Desreumeaux P, Huglo D, Hoorelbeke $\mathrm{A}$, Tonnel $\mathrm{AB}$, Wallaert $\mathrm{B}$. Increased intestinal permeability in bronchial asthma. J Allergy Clin Immunol. 1996;97(6):1173-8.

40 Majamaa H, Isolauri E. Evaluation of the gut mucosal barrier: evidence for increased antigen transfer in children with atopic eczema. J Allergy Clin Immunol. 1996;97(4):985-90.

41 Candela M, Rampelli S, Turroni S, Severgnini M, Consolandi C, De Bellis G, et al. Unbalance of intestinal microbiota in atopic children. BMC Microbiol. 2012;12(1):95.

42 Verhulst SL, Vael C, Beunckens C, Nelen V, Goossens H, Desager K. A longitudinal analysis on the association between antibiotic use, intestinal microflora, and wheezing during the first year of life. J Asthma. 2008;45(9): $828-32$.
43 Atarashi K, Tanoue T, Shima T, Imaoka A, Kuwahara T, Momose Y, et al. Induction of colonic regulatory $\mathrm{T}$ cells by indigenous Clostridium species. Science. 2011;331(6015): 337-41.

44 Atarashi K, Tanoue T, Oshima K, Suda W, Nagano Y, Nishikawa H, et al. Treg induction by a rationally selected mixture of Clostridia strains from the human microbiota. Nature. 2013;500(7461):232-6.

45 Noval Rivas M, Chatila TA. Regulatory T cells in allergic diseases. J Allergy Clin Immunol. 2016;138(3):639-52.

46 Manichanh C, Rigottier-Gois L, Bonnaud E, Gloux K, Pelletier E, Frangeul L, et al. Reduced diversity of faecal microbiota in Crohn's disease revealed by a metagenomic approach. Gut. 2006;55(2):205-11.

47 Simonyté Sjödin K, Hammarström ML, Rydén P, Sjödin A, Hernell O, Engstrand L, et al. Temporal and long-term gut microbiota variation in allergic disease: a prospective study from infancy to school age. Allergy. 2019; 74(1):176-85

48 Jiang S, Xie S, Lv D, Wang P, He H, Zhang T, et al. Alteration of the gut microbiota in Chinese population with chronic kidney disease. Sci Rep. 2017;7(1):2870

49 Scher JU, Ubeda C, Artacho A, Attur M, Isaac S, Reddy SM, et al. Decreased bacterial diversity characterizes the altered gut microbiota in patients with psoriatic arthritis, resembling dysbiosis in inflammatory bowel disease. Arthritis Rheumatol. 2015;67(1):128-39.
50 Hiippala K, Kainulainen V, Kalliomäki M, Arkkila P, Satokari R. Mucosal prevalence and interactions with the epithelium indicate commensalism of Sutterella spp. Front Microbiol. 2016;7:1706.

51 Ofek M, Hadar Y, Minz D. Ecology of root colonizing Massilia (Oxalobacteraceae). PLoS One. 2012;7(7):e40117.

52 Liu M, Koh H, Kurtz ZD, Battaglia T, PeBenito A, Li H, et al. Oxalobacter formigenes-associated host features and microbial community structures examined using the American Gut Project. Microbiome. 2017;5(1):108.

53 Pakarinen J, Hyvärinen A, Salkinoja-Salonen M, Laitinen S, Nevalainen A, Mäkelä MJ, et al. Predominance of Gram-positive bacteria in house dust in the low-allergy risk Russian Karelia. Environ Microbiol. 2008;10(12): 3317-25.

54 Wang Q, Li F, Liang B, Liang Y, Chen S, Mo $\mathrm{X}$, et al. A metagenome-wide association study of gut microbiota in asthma in UK adults. BMC Microbiol. 2018;18(1):114

55 Furusawa Y, Obata Y, Fukuda S, Endo TA Nakato G, Takahashi D, et al. Commensal microbe-derived butyrate induces the differentiation of colonic regulatory T cells. Nature. 2013;504(7480):446-50.

56 Chua HH, Chou HC, Tung YL, Chiang BL, Liao CC, Liu HH, et al. Intestinal dysbiosis featuring abundance of Ruminococcus gnavus associates with allergic diseases in infants. Gastroenterology. 2018;154(1):154-67.
Gut Microbiome of Adults with Allergic Rhinitis
Int Arch Allergy Immunol 2021;182:94-105 